\title{
Evaluation of a Three Compartment In Vitro Gastrointestinal Simulator Dissolution Apparatus to Predict In Vivo Dissolution
}

\author{
SUSUMU TAKEUCHI, ${ }^{1,2}$ YASUHIRO TSUME, ${ }^{1}$ GREGORY E. AMIDON, ${ }^{1}$ GORDON L. AMIDON ${ }^{1}$ \\ ${ }^{1}$ College of Pharmacy, University of Michigan, Ann Arbor, Michigan 48109-1065 \\ ${ }^{2}$ Pharmacokinetics group, Sawai Pharmaceutical Company, Ltd, Osaka 532-0003, Japan
}

Received 13 September 2013; revised 10 July 2014; accepted 14 July 2014

Published online 22 September 2014 in Wiley Online Library (wileyonlinelibrary.com). DOI 10.1002/jps.24112

\begin{abstract}
In vitro dissolution tests are performed for new formulations to evaluate in vivo performance, which is affected by the change of gastrointestinal (Gl) physiology, in the $\mathrm{Gl}$ tract. Thus, those environmental changes should be introduced to an in vitro dissolution test. Many studies have successfully shown the improvement of in vitro-in vivo correlations (IVIVC) by introducing those physiological changes into dissolution tests. The gastrointestinal simulator (GIS), a multicompartment in vitro dissolution apparatus, was developed to evaluate in vivo drug dissolution. A gastric-emptying rate along with transit rate are key factors to evaluate in vivo drug dissolution and, hence, drug absorption. Dissolution tests with the GIS were performed with Biopharmaceutical Classification System class I drugs at five different gastric-emptying rates in the fasted state. Computational models were used to determine in vivo gastric-emptying time for propranolol and metoprolol based on the GIS dissolution results. Those were compared with published clinical data to determine the gastric half-emptying time. In conclusion, the GIS is a practical tool to assess dissolution properties and can improve IVIVC. $\odot 2014$ Wiley Periodicals, Inc. and the American Pharmacists Association J Pharm Sci 103:3416-3422, 2014
\end{abstract}

Keywords: dissolution; ASD; GIS; gastric emptying; GastroPlus; in vitro/in vivo correlations (IVIVC); Gastrointestinal transit; In vitro models; Transit time; Dissolution rate

\section{INTRODUCTION}

Oral dosage forms disintegrate and dissolve in the gastrointestinal (GI) tract and are absorbed into the intestinal membrane. The drug concentration at the membrane determines the local absorption rate. The disintegration and dissolution rates of oral dosage forms depend on the physicochemical properties of the compound such as $\mathrm{pKa}$, crystalline energy, solubility, surface area, formulation, as well as the physiological properties in the GI tract. The Biopharmaceutical Classification System (BCS) class I and III drugs are highly soluble in the GI tract and their dissolution rates not likely to be a rate-limiting step in drug absorption. ${ }^{1}$ However, BCS class II drugs are categorized as low solubility and high permeability and the dissolution rate of BCS class II drugs will likely determine the drug absorption rate. Therefore, for the formulation development, it would be valuable for BCS Class II drugs to be able to predict in vivo performance.

USP dissolution tests apparatus 1 (rotating basket) and 2 (paddle) are routinely used for screening and optimizing formulations and to assure product consistency and quality. Often, however, the test conditions do not reflect the in vivo environment of the GI tract. The physiological environment such as buffer species, buffer capacity, $\mathrm{pH}$, bile salt, liquid volume, and gastric-emptying rate (motility) are expected to influence in vivo dissolution that affects the drug absorption. ${ }^{2-5}$ The incorporation of physiologically relevant environmental factors into an in vitro dissolution apparatus would likely lead to more accurate in vivo dissolution predictions. Numerous in vitro dissolution procedures have been proposed, such as using biorelevant

Correspondence to: Gordon L. Amidon (Telephone: +734-764-2440; Fax: +734-763-6423; E-mail: glamidon@med.umich.edu)

Journal of Pharmaceutical Sciences, Vol. 103, 3416-3422 (2014)

(C) 2014 Wiley Periodicals, Inc. and the American Pharmacists Association media, a two-phase dissolution apparatus, a transfer model, and also the artificial stomach-duodenum (ASD) apparatus. Biorelevant media (FaSSGF, FaSSIF, FeSSIF, and bicarbonate buffers) have been introduced to improve IVIVC as these media more closely reflect the in vivo lumenal fluid. ${ }^{6-8}$ As it consists of both the drug dissolution component (aqueous phase) and the absorption component (organic phase), the two-phase dissolution apparatus can provide valuable insight to predict in vivo performance. ${ }^{9}$ The transfer model and the ASD can represent the environmental changes through the GI tract, such as liquid volume and $\mathrm{pH}$, which can influence the amount of dissolved drug.

Kostewicz et al. ${ }^{10}$ utilized the transfer model for poorly soluble weak base drugs to predict in vivo precipitation rate with changing transit rate from the stomach to the duodenum. The precipitation rates in in vitro tests were evaluated in the fasted and the fed states. On the basis of these results, the transfer model better represented in vivo performance for the drugs studied. Carino and coworkers ${ }^{11,12}$ developed the ASD apparatus that is the two-compartment dissolution system consisting of chambers representing the stomach and duodenum. ASD dissolution tests of carbamazepine were performed to understand in vivo dissolution of different crystal forms of carbamazepine in the fasted and the fed states. The ASD accurately predicted drug absorption in dogs for the different crystal forms. The ASD system has successfully demonstrated in vivo phenomena and, therefore, supports the usefulness of the two-compartment dissolution system to improve IVIVC.

Because the residence time in the human jejunum and ileum is much longer than the transit time of the human duodenum ( $\sim 30 \mathrm{~min})$, most drugs will be absorbed along the small intestine. Thus, drug concentration in the jejunal chamber, rather than simply in the duodenal chamber alone, was considered important for in vitro dissolution to predict drug absorption. ${ }^{13,14}$ 
Weak basic drugs, for example, will be more readily dissolved in the acidic condition and then may precipitate or remain as a supersaturated solution upon entering the duodenum because of the higher lumenal $\mathrm{pH} \cdot{ }^{15,16}$ Supersaturation will likely enhance the bioavailability of lipophilic weak basic drugs. ${ }^{17,18}$ The supersaturation and precipitation rate will be affected by the transit rate, because of the changes of drug concentration, buffer capacity, and $\mathrm{pH}$ that occur in association with the transit rate. Hence, the determination of transit rate from the gastric chamber to the duodenal chamber is an important factor in the multicompartment dissolution apparatus for the evaluation of in vivo dissolution.

In this study, we developed an apparatus called gastrointestinal simulator (GIS), based on the ASD system of Carino and coworkers, ${ }^{11,12}$ which consists of the gastric, duodenal, and jejunal chamber, and we determined the gastric-emptying time and transit time required to simulate in vivo performance of propranolol and metoprolol. The dissolution tests of propranolol and metoprolol were performed with the GIS at five different transfer rates representing the gastric-emptying rate. The predicted pharmacokinetics parameters were obtained by GastroPlus ${ }^{\mathrm{TM}}$ based on dissolution results. Those results were compared with the clinical data to determine the appropriate transfer rate needed to best simulate the gastric half-emptying time in the GIS. This system may be able to perform better IVIVC by monitoring the dissolution rate, the dissolved drug concentration, and the potential measurement of drug precipitation and supersaturation in these chambers.

\section{MATERIALS AND METHODS}

\section{Materials}

Metoprolol tablets (100 mg) and propranolol tablets (40 mg) were obtained from Ingenus Pharmaceuticals (Orlando, Florida) and PLIVA (Sarajevo, Bosnia and Herzegovina), respectively. Metoprolol tartrate, propranolol hydrochloride, hydrochloric acid, sodium phosphate dibasic, and sodium chloride were obtained from Sigma-Aldrich Chemical Company (St. Louis, Missouri). Orthophosphoric acid 85\%, trifluoroacetic acid, methanol, and acetonitrile were obtained from Fisher Scientific Inc. (Pittsburgh, Pennsylvania). All chemicals were of analytical grade or of HPLC grade.

\section{Gastrointestinal Simulator}

The diagram of the GIS is shown in Figure 1a and consists of three dissolution chambers representing the stomach, the duodenum, and the jejunum. Four precision pumps are utilized to simulate gastric fluid secretion, duodenal fluid secretion, transfer of gastric chamber contents to the duodenal chamber, and also to transfer duodenal contents to the jejunal chamber. All pump speeds can be adjusted. The gastric and duodenal fluids were maintained at $37^{\circ} \mathrm{C}$ in the water bath, and $\mathrm{pH}$ probes were placed in the gastric and duodenal chambers to monitor $\mathrm{pH}$ at $60 \mathrm{~s}$ intervals. The paddles in the gastric and duodenal chambers were appropriately controlled to simulate relevant gastric and duodenal motility. The jejunal chamber was stirred at a

a

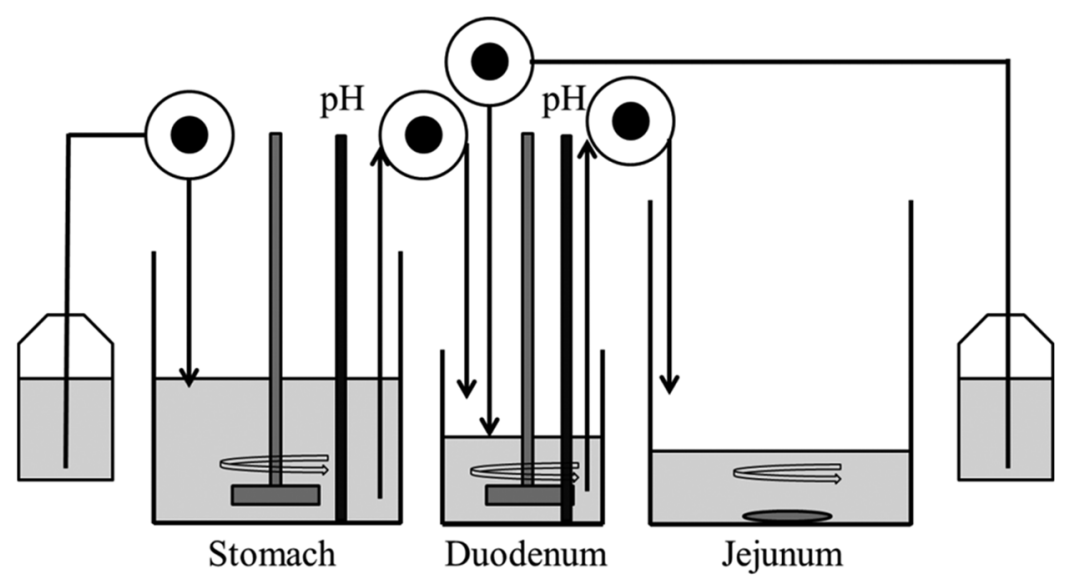

b

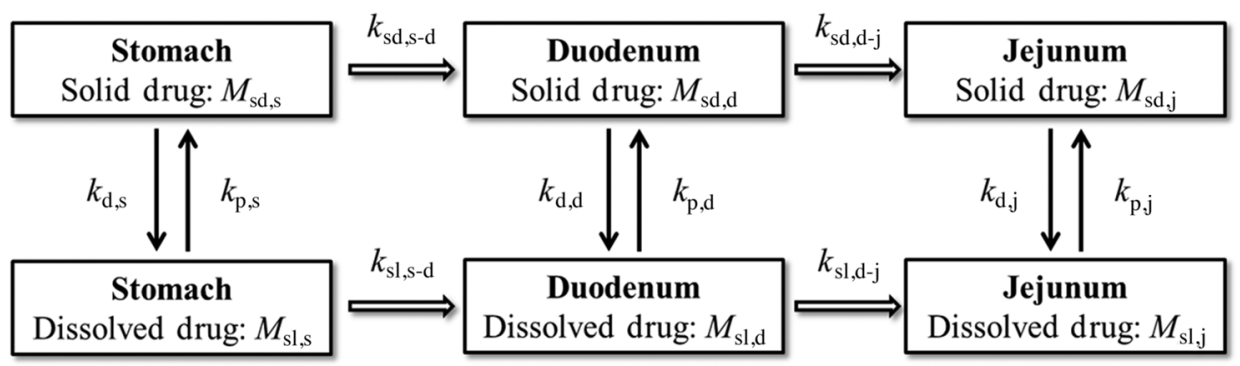

Figure 1. The diagram of GIS (a). The GIS dissolution model (b). 
constant speed. Samples were taken from each chamber at a specific time, and supernatant was diluted with $\mathrm{H}_{2} \mathrm{O}-$ methanol $(1 / 1, v / v)$ after centrifugation $(2000 g, 20 \mathrm{~s})$. Dissolved drug concentration was determined by HPLC analysis.

\section{GIS Condition, Process, and Theory}

The GIS system was designed to represent the physiological conditions of the human GI tract in the fasted state. The test conditions were as follows. The gastric chamber was filled with $50 \mathrm{~mL}$ of $0.01 \mathrm{~N} \mathrm{HCl}$ to represent the gastric fluid and $250 \mathrm{~mL}$ of water to represent the dose volume. The duodenal chamber was filled with $50 \mathrm{~mL}$ of $0.05 \mathrm{M}$ sodium phosphate buffer ( $\mathrm{pH}$ 6.5) to represent duodenal fluid, and the jejunal chamber was left empty. During dissolution testing, simulated gastric fluid $(0.01 \mathrm{~N} \mathrm{HCl})$ and simulated duodenal fluid (0.1 M sodium phosphate buffer, $\mathrm{pH}$ 6.5) were pumped into each chamber at $1 \mathrm{~mL} / \mathrm{min}$. The tablet was placed into the gastric chamber to start the dissolution test. The gastric contents, both dissolved and undissolved material, were moved to the duodenal chamber at the first-order rate that could be set to any value as the gastric half-emptying time. Additionally, the duodenal contents were moved to the jejunal chamber with the same rate as the gastric half-emptying rate and the duodenal fluid secretion rate. The fluid volume in the duodenal chamber thus was maintained at $50 \mathrm{~mL}$ during dissolution testing.

Figure 1b shows the various dynamic processes in the GIS chambers. The test tablet is introduced into the gastric chamber where disintegration can occur. Immediate-release dosage forms are expected to disintegrate and potentially dissolve to some extent in the gastric chamber. However, dissolved drug may be precipitated in the gastric chamber depending on the physicochemical properties of the drug. The dissolved drugs, solid drug particles, and excipients present in the gastric chamber are transferred to the duodenal chamber. The mathematical models representing these processes are shown in Eqs. (1) and (2) as follows:

$$
\begin{gathered}
\frac{\mathrm{d} M_{\mathrm{sd}, \mathrm{s}}}{\mathrm{d} t}=-k_{\mathrm{d}, \mathrm{s}} M_{\mathrm{sd}, \mathrm{s}}-k_{\mathrm{sd}, \mathrm{s}-\mathrm{d}} M_{\mathrm{sd}, \mathrm{s}}+k_{\mathrm{p}, \mathrm{s}} M_{\mathrm{sl}, \mathrm{s}} \\
\frac{\mathrm{d} M_{\mathrm{sl}, \mathrm{s}}}{\mathrm{d} t}=k_{\mathrm{d}, \mathrm{s}} M_{\mathrm{sd}, \mathrm{s}}-k_{\mathrm{sl}, \mathrm{s}-\mathrm{d}} M_{\mathrm{sl}, \mathrm{s}}-k_{\mathrm{p}, \mathrm{s}} M_{\mathrm{sl}, \mathrm{s}}
\end{gathered}
$$

where $M_{\mathrm{sd}, \mathrm{s}}$ and $M_{\mathrm{sl}, \mathrm{s}}$ are the amounts of solid drug (sd) and dissolved drug in solution (sl) in the gastric chamber, $k_{\mathrm{d}, \mathrm{s}}$ and $k_{\mathrm{p}, \mathrm{s}}$ are the dissolution rate constant and the precipitation rate constant in the gastric chamber, respectively. The transfer rate constants for solid drug and solution drug from the gastric chamber to the duodenal chamber are $k_{\mathrm{sd}, \mathrm{s}-\mathrm{d}}$ and $k_{\mathrm{sl}, \mathrm{s}-\mathrm{d}}$. The expressions for dissolution and precipitation rates are approximate mathematical expressions, included here for more symbolic purposes. They assume constant, first-order processes that are not affected by factors such as drug saturation solubility or variable $\mathrm{pH}$ in the bulk fluid and at the surface of the drug particles. These approximations are likely suitable for this particular study, because of the high solubility of propranolol and metoprolol. The development of mechanistic models for dissolution and precipitation rates in the GIS system was not within the scope of the current study.
In the duodenal chamber, weak acid drugs might be dissolved, whereas dissolved weak basic drugs might be precipitated. In the jejunal chamber, dissolution and precipitation proceed depending on the physicochemical properties of drugs. The amount of solid drug and dissolved drug in the duodenum and jejunum chamber is shown in Eqs. (3)-(6) as follows:

(Duodenal chamber)

$$
\begin{gathered}
\frac{\mathrm{d} M_{\mathrm{sd}, \mathrm{d}}}{\mathrm{d} t}=k_{\mathrm{sd}, \mathrm{s}-\mathrm{d}} M_{\mathrm{sd}, \mathrm{s}}-k_{\mathrm{d}, \mathrm{d}} M_{\mathrm{sd}, \mathrm{d}}-k_{\mathrm{sd}, \mathrm{d}-\mathrm{j}} M_{\mathrm{sd}, \mathrm{d}}+k_{\mathrm{p}, \mathrm{d}} M_{\mathrm{sl}, \mathrm{d}} \\
\frac{\mathrm{d} M_{\mathrm{sl}, \mathrm{d}}}{\mathrm{d} t}=k_{\mathrm{sl}, \mathrm{s}-\mathrm{d}} M_{\mathrm{sl}, \mathrm{s}}+k_{\mathrm{d}, \mathrm{d}} M_{\mathrm{sd}, \mathrm{d}}-k_{\mathrm{sl}, \mathrm{d}-\mathrm{j}} M_{\mathrm{sl}, \mathrm{d}}-k_{\mathrm{p}, \mathrm{d}} M_{\mathrm{sl}, \mathrm{d}}
\end{gathered}
$$

(Jejunal chamber)

$$
\begin{aligned}
& \frac{\mathrm{d} M_{\mathrm{sd}, \mathrm{j}}}{\mathrm{d} t}=k_{\mathrm{sd}, \mathrm{d}-\mathrm{j}} M_{\mathrm{sd}, \mathrm{d}}-k_{\mathrm{d}, \mathrm{j}} M_{\mathrm{sd}, \mathrm{j}}+k_{\mathrm{p}, \mathrm{j}} M_{\mathrm{sl}, \mathrm{j}} \\
& \frac{\mathrm{d} M_{\mathrm{sl}, \mathrm{j}}}{\mathrm{d} t}=k_{\mathrm{sl}, \mathrm{d}-\mathrm{j}} M_{\mathrm{sl}, \mathrm{d}}+k_{\mathrm{d}, \mathrm{j}} M_{\mathrm{sd}, \mathrm{j}}-k_{\mathrm{p}, \mathrm{j}} M_{\mathrm{sl}, \mathrm{j}}
\end{aligned}
$$

where $M_{\mathrm{sd}, \mathrm{d}}, M_{\mathrm{sl}, \mathrm{d}}, M_{\mathrm{sd}, \mathrm{j}}$, and $M_{\mathrm{sl}, \mathrm{j}}$ are the amounts of solid drug and dissolved drug in the duodenal and jejunal chambers, respectively. $k_{\mathrm{d}, \mathrm{d}}, k_{\mathrm{p}, \mathrm{d}}, k_{\mathrm{d}, \mathrm{j}}$, and $k_{\mathrm{p}, \mathrm{j}}$ are the dissolution rate constants and the precipitation rate constants in the duodenal and jejunal chambers, respectively. The transfer rate constants for solid drug and dissolved drug from the duodenal chamber to the jejunal chamber are $k_{\mathrm{sd}, \mathrm{d}-\mathrm{j}}$ and $k_{\mathrm{sl}, \mathrm{d}-\mathrm{j}}$.

In this study, the GIS was used with five different gastric half-emptying times $\left(t_{1 / 2}=5,10,15,20\right.$, and $30 \mathrm{~min}$ ). Oral drug absorption and pharmacokinetics parameters were predicted by GastroPlus ${ }^{\mathrm{TM}}$ (version 6.0; SimulationPlus, Inc., Lancaster, California). The pharmacokinetics parameters $\left(C_{\max }, T_{\max }\right.$, and AUC) between clinical data and in silico results were then compared to determine the gastric halfemptying time in the GIS that best simulated in vivo results.

\section{GastroPlus $^{\mathrm{TM}}$ Simulation}

The physicochemical and biopharmaceutical properties of propranolol and metoprolol used in the GastroPlus ${ }^{\mathrm{TM}}$ prediction are presented in Table $1 .^{19-26}$ The dissolution profiles with the GIS were created by adding together the dissolved drug in both the duodenal and jejunal chambers. The absorption was predicted by GastroPlus ${ }^{\mathrm{TM}}$ with those dissolution profiles for propranolol and metoprolol. No drug absorption from the stomach was assumed in this set of predictions, and the dosage form drug release was simulated as a controlled-release tablet in the GastroPlus ${ }^{\mathrm{TM}}$ based on the dissolution profiles of propranolol and metoprolol obtained with the GIS (Figs. 2e and 4e). Virtual trials were performed ( $n=100)$ on each gastric half-emptying time to obtain plasma concentration curves and pharmacokinetics parameters.

\section{RESULTS}

\section{Propranolol}

Figures $2 \mathrm{a}$ and $2 \mathrm{~b}$ present the mean dissolved drug concentration-time profiles in each chamber with the fastest 
Table 1. Chemical/Physiological/Pharmacological Parameters of Propranolol and Metoprolol for GastroPlus ${ }^{\mathrm{TM}}$ Simulation

\begin{tabular}{lcc}
\hline & Propranolol & Metoprolol \\
\hline MW & 259.3 & 267.4 \\
Dose $(\mathrm{mg})$ & $80^{a}$ & $100^{a}$ \\
Dose volume $(\mathrm{mL})$ & 250 & 250 \\
Solubility $(\mathrm{mg} / \mathrm{mL})$ & $33^{b}$ & $16.9^{c}$ \\
$\log P$ & $2.65^{d}$ & $1.72^{d}$ \\
pKa & $9.5^{e}$ & $9.7^{b}$ \\
Human $P_{\text {eff }}\left(\times 10^{-4} \mathrm{~cm}^{2} / \mathrm{s}\right)$ & $2.9^{d}$ & $1.5^{a}$ \\
Body weight $(\mathrm{kg})$ & 70 & 70 \\
$V_{\mathrm{c}}(\mathrm{L} / \mathrm{kg})$ & $4.2^{f}$ & $5.2^{g}$ \\
Total clearance $(\mathrm{L} / \mathrm{h})$ & $65^{h}$ & $83^{g}$ \\
\hline
\end{tabular}

$V_{\mathrm{c}}$, volume of central compartment;

${ }^{a}$ Ref. 19.

${ }^{b}$ Ref. 20 .

${ }^{c}$ Ref. 21.

${ }^{d}$ Ref. 22 .

${ }^{e}$ Ref. 23.

${ }^{\text {Ref. } 24}$

gef. 25

${ }^{h}$ Ref. 26.

and slowest gastric half-emptying times, 5 or $30 \mathrm{~min}$, in this set of experiments. Two $40 \mathrm{mg}$ propranolol tablets (maximum dose, $80 \mathrm{mg}$ ) were placed into the gastric chamber to start the GIS dissolution study. Maximum dissolved drug concentration $\left(c_{\max }\right)$ in each chamber was influenced by the gastric half-emptying time and transit time; $c_{\max }$ ratios between 5 and 30 min gastric half-emptying time $\left(c_{\max } 5 \mathrm{~min} / c_{\max } 30 \mathrm{~min}\right)$ were increased by $15.5 \%$ (the gastric chamber), $30.2 \%$ (the duodenal chamber), and $13.4 \%$ (the jejunal chamber).

Figures $2 \mathrm{c}$ and $2 \mathrm{~d}$ present the mean dissolved drug (\%)-time profiles in each chamber with the fastest and slowest gastric half-emptying times, 5 or $30 \mathrm{~min}$, in this set of experiments. Comparing the results of the GIS dissolution tests between 5 and 30 min gastric half-emptying time, the time of maximum dissolved drug $\left(t_{\max }\right)$ was delayed from 2.5 to 5 min in the gastric chamber, and from 12.5 to $25 \mathrm{~min}$ in the duodenal chamber. After achieving $t_{\max }$ in respective chambers, the amounts of dissolved drug were steadily decreased as the contents were transferred to the next chamber. In contrast, the amounts of the dissolved drug in the jejunal chamber were steadily increased because of transfer from the duodenal chamber.

With five different gastric half-emptying times, the sum of the dissolved drug in the duodenal and jejunal chambers was calculated as shown in Figure 2e. The gastric half-emptying time influenced the drug amount in the duodenal and jejunal chambers. However, the gastric half-emptying time did not influence the dissolution rate of propranolol tablets, which were completely dissolved within 7.5 min (Fig. 2f).

Figure 3 shows that the predicted oral absorption of propranolol by GastroPlus ${ }^{\mathrm{TM}}$ using the dissolution profiles from the GIS along with human clinical data. Shifts in the plasma

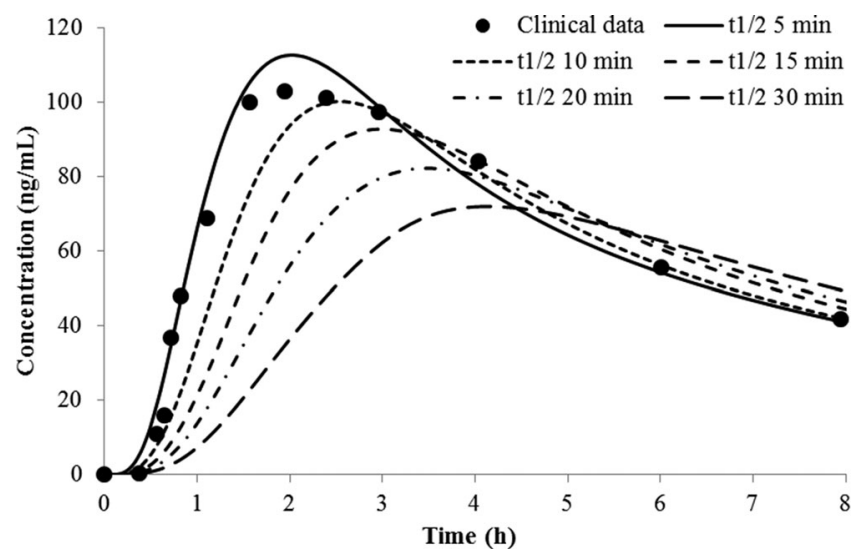

Figure 3. Comparison of plasma concentration-time profiles for propranolol tablets between predicted data by GastroPlus ${ }^{\text {TM }}$ and clinical data from Eddington et al. ${ }^{27}$
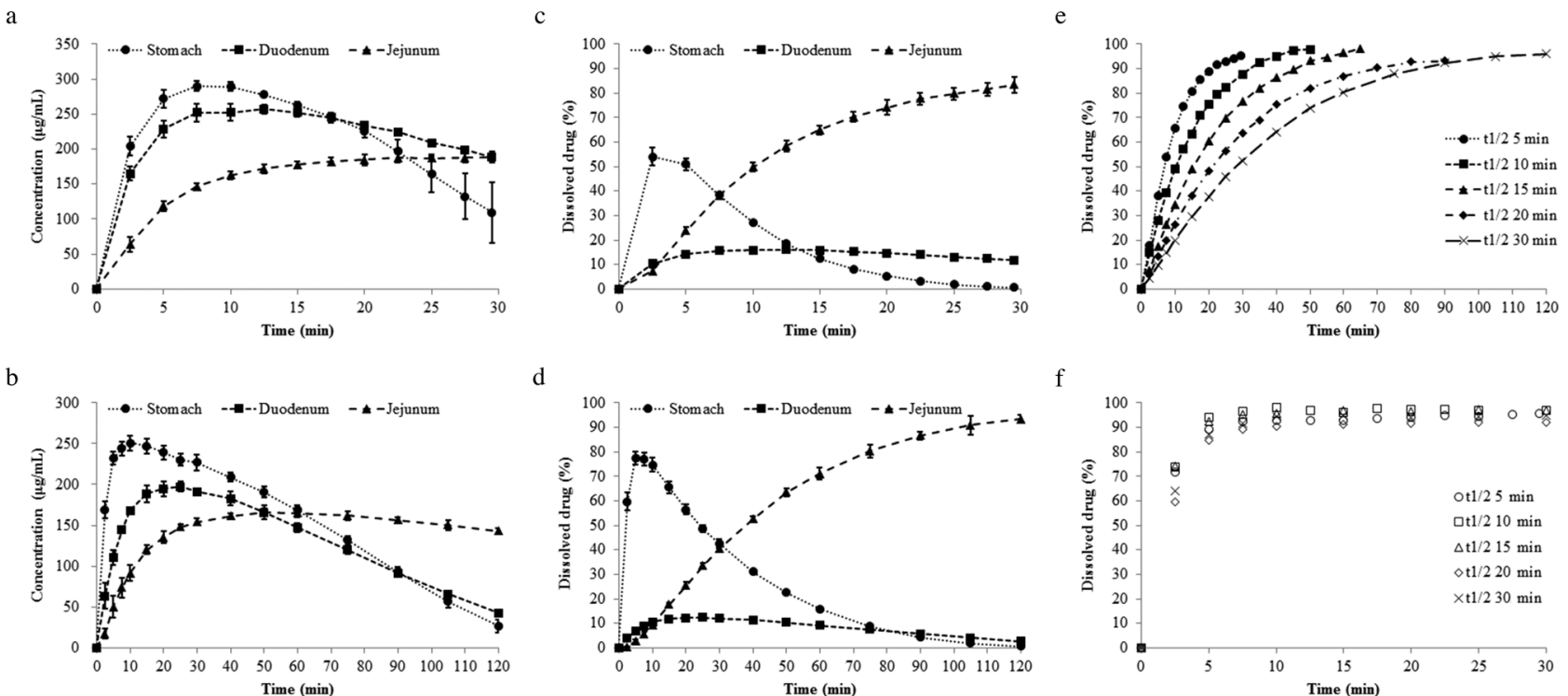

Figure 2. The dissolved drug concentration-time profiles of propranolol in the gastric, duodenal, and jejunal chambers with the fastest and slowest gastric half-emptying time: 5 (a) and $30 \mathrm{~min}$ (b). Dissolved drug-time profiles of propranolol in the gastric, duodenal, and jejunal chambers with 5 (c) and $30 \mathrm{~min}(\mathrm{~d})$. The sum of dissolved drug-time profiles of propranolol in the duodenal and jejunal chambers (e). The sum of dissolved drug-time profiles of propranolol in the gastric, duodenal, and jejunal chambers (f). Each data point represents mean \pm SD $(n=3)$. 
Table 2. Mean Pharmacokinetics Parameters for Propranolol and Metoprolol Simulated by GastroPlus ${ }^{\mathrm{TM}}$

\begin{tabular}{|c|c|c|c|c|c|c|}
\hline \multirow{2}{*}{$\begin{array}{l}\text { Gastric Half-Emptying Time } \\
\left(t_{1 / 2}, \min \right)\end{array}$} & \multicolumn{3}{|c|}{ Propranolol } & \multicolumn{3}{|c|}{ Metoprolol } \\
\hline & $C_{\max }(\mathrm{ng} / \mathrm{mL})$ & $T_{\max }(\mathrm{h})$ & $\mathrm{AUC}_{\infty}(\mathrm{ng} \mathrm{h} / \mathrm{mL})$ & $C_{\max }(\mathrm{ng} / \mathrm{mL})$ & $T_{\max }(\mathrm{h})$ & $\mathrm{AUC}_{\infty}(\mathrm{ng} \mathrm{h} / \mathrm{mL})$ \\
\hline 5 & 110.8 & 2.0 & 858.6 & 89.6 & 1.5 & 421.5 \\
\hline 10 & 98.6 & 2.5 & 827.3 & 79.5 & 2.0 & 424.5 \\
\hline 15 & 91.4 & 3.0 & 824.4 & 69.1 & 2.5 & 402.0 \\
\hline 20 & 81.0 & 3.5 & 803.0 & 60.7 & 3.1 & 388.3 \\
\hline 30 & 70.9 & 4.1 & 779.1 & 50.0 & 3.9 & 374.7 \\
\hline Clinical data & $109.5^{a}$ & $2.1^{a}$ & $821.6^{a}$ & $89.4^{b}$ & $1.6^{b}$ & $444.6^{b}$ \\
\hline
\end{tabular}

\footnotetext{
${ }^{a}$ Ref. 27.

${ }^{b}$ Ref. 28 .

Virtual trials $(n=100)$.
}

concentration-time curves were observed because of slow gastric half-emptying time. The plasma concentration-time profile with 5 min gastric half-emptying time displayed a plasma concentration curve very similar to clinical data. These results indicate that the gastric half-emptying time in the GIS should be between 5 and $10 \mathrm{~min}$ (Table 2).

\section{Metoprolol}

Figures $4 \mathrm{a}$ and $4 \mathrm{~b}$ present the mean dissolved drug concentration-time profiles in each chamber with the fastest and slowest gastric half-emptying times, 5 or $30 \mathrm{~min}$, in this set of experiments. One $100 \mathrm{mg}$ metoprolol tablet (maximum dose, $100 \mathrm{mg}$ ) was placed into the gastric chamber to start the GIS dissolution study. The $c_{\max }$ in each chamber was influenced by the gastric half-emptying time and transit time; $c_{\max }$ ratios between 5 and $30 \mathrm{~min}$ gastric half-emptying time $\left(c_{\max } 5 \mathrm{~min} / c_{\max }\right.$ $30 \mathrm{~min}$ ) were increased by $148 \%$ and $92.3 \%$ in the gastric and duodenal chambers, but were decreased by $6.3 \%$ in the jejunal chamber.

Figures 4c and 4d present the mean dissolved drug (\%)-time profiles in each chamber with the fastest and slowest gastric half-emptying times, 5 or $30 \mathrm{~min}$, in this set of experiments. Comparing the results of the GIS dissolution tests between 5 and 30 min gastric half-emptying time, $t_{\max }$ was delayed from 12.5 to $15 \mathrm{~min}$ in the gastric chamber, and from 25 to $30 \mathrm{~min}$ in the duodenal chamber. After achieving $t_{\max }$ in both chambers, the amounts of dissolved drug were steadily decreased as the contents were transferred to the next chamber. In contrast, the amount of the dissolved drug in the jejunal chamber steadily increased because of transfer from the duodenal chamber.

With five different gastric half-emptying times, the sum of the dissolved drug in the duodenal and jejunal chambers was calculated as shown in Figure 4e. The gastric half-emptying time influenced the drug amount in the duodenal and jejunal chambers. However, the gastric half-emptying time did not influence the dissolution rate of metoprolol tablets, which were completely dissolved within 20 min (Fig. 4f).

Figure 5 shows that the predicted oral absorption of metoprolol by GastroPlus ${ }^{\text {TM }}$ using the dissolution profiles from the GIS along with human clinical data. Shifts of plasma concentration-time curves were observed because of slow gastric half-emptying time. Plasma concentration-time profile a

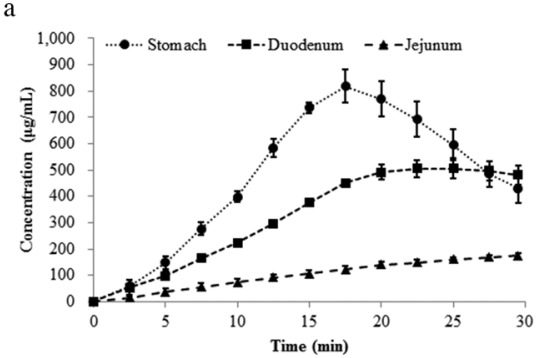

b

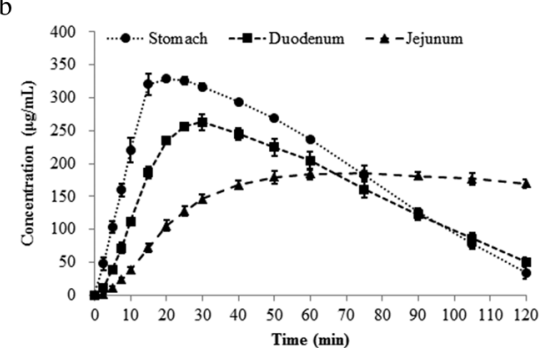

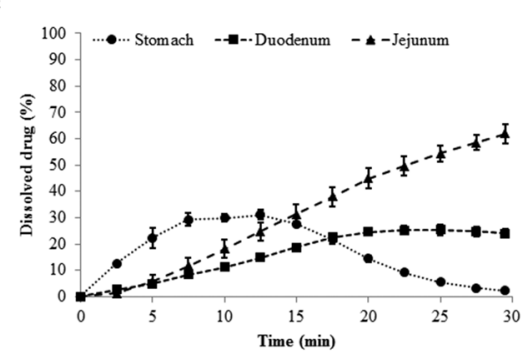

d

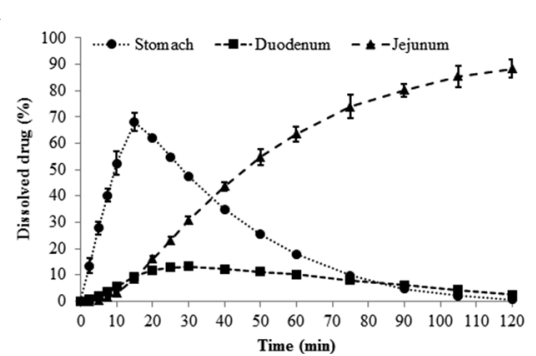

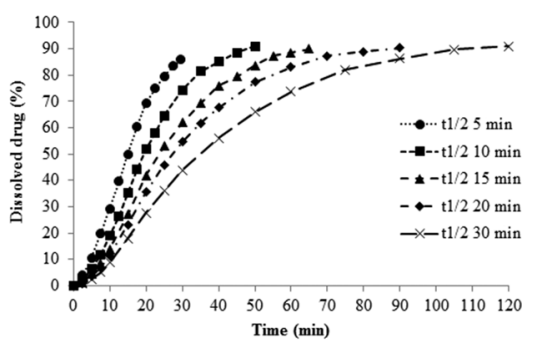

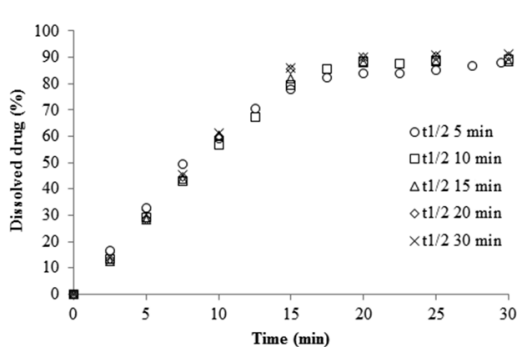

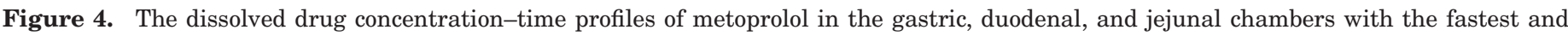

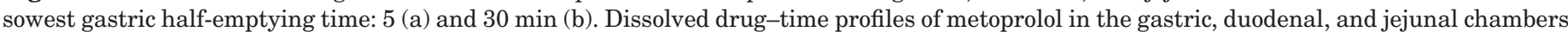

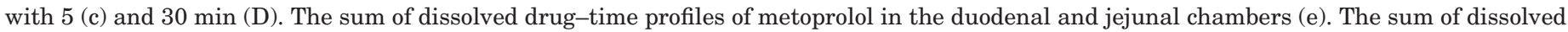
drug-time profiles of metoprolol in the gastric, duodenal, and jejunal chambers (f). Each data point represents mean $\pm \mathrm{SD}(n=3)$. 


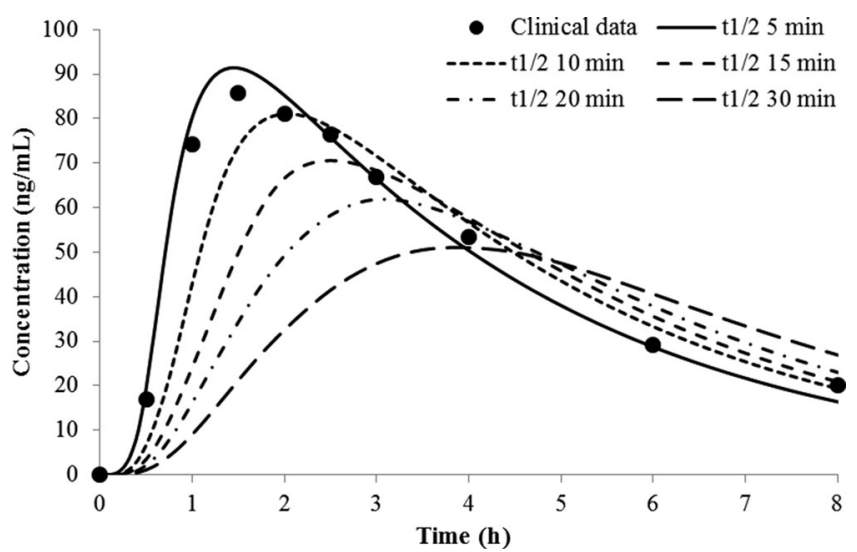

Figure 5. Comparison of plasma concentration-time profiles for metoprolol tablets between predicted data by GastroPlus ${ }^{\mathrm{TM}}$ and clinical data from Rekhi et al. ${ }^{28}$

with 5 min gastric half-emptying time displayed similar plasma concentration curves to clinical data. These results are consistent with those observed for propranolol (Table 2).

\section{DISCUSSION}

The gastric-emptying time and the transit time in the GI tract are important factors to predict in vivo dissolution because the gastric-emptying time can affect fluid volume in stomach and the transit time can affect drug dissolution and drug absorption in the small intestine. However, most in vitro dissolution apparatuses do not consider gastric-emptying time and intestinal transit or residence time. The incorporation of these factors into an in vitro dissolution apparatus can lead to better predictions of in vivo dissolution. Therefore, it is necessary to develop a dissolution apparatus, the GIS, to simulate gastric emptying to predict better in vivo dissolution. In the human fasted state, it has been reported that liquids gastric half-emptying time was $4.2,{ }^{29} 12,{ }^{30}$ and $15.8 \mathrm{~min} .{ }^{31}$ In this set of dissolution studies, five different gastric emptying half-times $(5,10,15,20$, and 30 min) were tested with the GIS, and pharmacokinetics parameters were obtained by GastroPlus ${ }^{\mathrm{TM}}$ based on the dissolution results with the GIS. Those pharmacokinetics parameters were compared with human clinical data.

As propranolol and metoprolol are highly soluble over the physiological $\mathrm{pH}$ range and also highly permeable in the entire small intestine, it is likely that gastric-emptying rate itself is the rate-limiting step to appearance of drug in the bloodstream. As shown in Figures $2 \mathrm{f}$ (propranolol) and $4 \mathrm{f}$ (metoprolol), total dissolution rate in the entire GIS system was not influenced by gastric emptying or transit rate because both drugs are of high solubility. However, the appearance of dissolved drug in the duodenal and jejunal chambers was delayed for both drugs with an increase in gastric-emptying half-time, which can be attributed simply to the slower gastric emptying.

Although direct comparison of experimental and predicted results requires direct measurement of drug concentrations in solution in the intestinal fluid, a qualitative comparison can be made based on average plasma levels in the literature. The comparison of pharmacokinetics parameters and plasma concentration curves between human clinical data and in silico data can suggest the suitable transit time for the GIS to pre- dict in vivo dissolution. In silico software revealed that the time of maximum plasma concentration $\left(T_{\max }\right)$ and maximum plasma concentration $\left(C_{\max }\right)$ were influenced by the gastric halfemptying time. By comparing these pharmacokinetics parameters with human clinical data, the gastric half-emptying time between 5 and $10 \mathrm{~min}$ for the GIS studies provided a good match to human in vivo systemic availability (and presumably the luminal fluid dissolution) in the fasted state (Figs. 3 and 5 ; Table 2). This relatively rapid estimate of gastric emptying time using the GastroPlus ${ }^{\mathrm{TM}}$ simulation for these rapidly disintegrating and dissolving IR dosage forms is consistent with one study that indicated a more rapid emptying of liquids from the stomach. ${ }^{29}$ However, it is shorter than other measurements indicating a gastric half-emptying time of about 15 min. ${ }^{13}$

In this study, dissolution tests with the GIS were performed for propranolol and metoprolol. Propranolol and metoprolol exhibited different times for complete dissolution (propranolol: $7.5 \mathrm{~min}$; metoprolol: $20 \mathrm{~min}$ ) (Figs. $2 \mathrm{f}$ and $4 \mathrm{f}$ ). With a gastric half-emptying time of $5 \mathrm{~min}$, this difference clearly influenced drug concentration in each chamber, but this difference did not influence drug concentration in the duodenal and jejunal chamber with a gastric half-emptying time of $30 \mathrm{~min}$ (Figs. 2 and 4 ). Comparing the concentration profiles ( $c_{\max }$ concentration/dose ratio) between propranolol and metoprolol with the gastric half-emptying time at $5 \mathrm{~min}$, metoprolol exhibited $126 \%$ and $57 \%$ higher $c_{\max }$ in the gastric and duodenal chambers than propranolol. However, metoprolol exhibited $26 \%$ lower $c_{\max }$ in the jejunal chamber than propranolol. Furthermore, comparing $t_{\max }$ between propranolol and metoprolol with the gastric emptying time at $5 \mathrm{~min}$, a $t_{\max }$ difference was observed in each chamber, propranolol (2.5 min) versus metoprolol (12.5 min) in the gastric chamber, and propranolol (12.5 min) versus metoprolol ( $25 \mathrm{~min}$ ) in the duodenal chamber. As these results show, the GIS can evaluate disintegration time and dissolution rate in each chamber depending on test drugs. However, the short gastric-emptying time suggested requiring further direct measurement in the luminal intestinal fluid.

The GIS can not only assess $c_{\max }$ and $t_{\max }$, but can also evaluate the in vivo dissolution profile. For example, Carino et al. ${ }^{11}$ performed the ASD, of which the GIS is an adaption with a jejunal compartment, to estimate bioavailability of carbamazepine (BCS class II drug) that has three different crystal forms. Dissolution profiles of different carbamazepine crystal forms in the duodenal chamber were compared with oral bioavailability of those crystal forms in dogs. The results showed an excellent correlation between the ASD dissolution results and dog bioavailability. For BCS class II weak basic drugs, the precipitation rate and supersaturation in the GI tract will significantly affect oral bioavailability. The GIS allows these phenomena in the duodenal and jejunal chambers to be observed and measured, in a less complex environment than the luminal intestinal fluid, and can provide valuable information with which to predict in vivo dissolution. Thus, the GIS appears to be a very useful tool for the development of oral drug formulations.

\section{CONCLUSION}

The dissolution results with the GIS system provide a convenient means of quantitating drug concentration in the chambers representing the stomach, duodenum, and jejunum that directly impact in vivo drug absorption. This in vitro GI com- 
partment concentration is the information needed for the prediction of oral drug absorption. The gastric half-emptying time in the fasted state was determined in these studies as a part of the GIS development. This is a significant factor for a multicompartment dissolution apparatus in the prediction of in vivo dissolution. In this study, the gastric half-emptying time for the GIS was observed to be between 5 and $10 \mathrm{~min}$, and although it is in general agreement with previous reports of the gastric emptying of liquids, ${ }^{29,30}$ it is shorter than other reported value. ${ }^{13}$ This requires direct confirmation by measuring luminal fluid concentrations in the future.

\section{REFERENCES}

1. Amidon GL, Lennernas H, Shah VP, Crison JR. 1995. A theoretical basis for a biopharmaceutic drug classification: The correlation of in vitro drug product dissolution and in vivo bioavailability. Pharm Res 12(3):413-420.

2. Mooney KG, Mintun MA, Himmelstein KJ, Stella VJ. 1981. Dissolution kinetics of carboxylic acids II: Effect of buffers. J Pharm Sci 70(1):22-32

3. Fallingborg J. 1999. Intraluminal $\mathrm{pH}$ of the human gastrointestinal tract. Dan Med Bull 46(3):183-196.

4. Vertzoni M, Fotaki N, Kostewicz E, Stippler E, Leuner C, Nicolaides E, Dressman J, Reppas C. 2004. Dissolution media simulating the intralumenal composition of the small intestine: Physiological issues and practical aspects. J Pharm Pharmacol 56(4):453462.

5. Tsume Y, Langguth $P$, Garcia-Arieta A, Amidon GL. 2012. In silico prediction of drug dissolution and absorption with variation in intestinal $\mathrm{pH}$ for BCS class II weak acid drugs: Ibuprofen and ketoprofen. Biopharm Drug Dispos 33(7):366-377.

6. Galia E, Nicolaides E, Horter D, Lobenberg R, Reppas C, Dressman JB. 1998. Evaluation of various dissolution media for predicting in vivo performance of class I and II drugs. Pharm Res 15(5):698-705.

7. Jantratid E, Janssen N, Reppas C, Dressman JB. 2008. Dissolution media simulating conditions in the proximal human gastrointestinal tract: An update. Pharm Res 25(7):1663-1676.

8. Sheng JJ, McNamara DP, Amidon GL. 2009. Toward an in vivo dissolution methodology: A comparison of phosphate and bicarbonate buffers. Mol Pharm 6(1):29-39.

9. Mudie DM, Shi Y, Ping H, Gao P, Amidon GL, Amidon GE. 2012. Mechanistic analysis of solute transport in an in vitro physiological two-phase dissolution apparatus. Biopharm Drug Dispos 33(7):378402.

10. Kostewicz ES, Wunderlich M, Brauns U, Becker R, Bock T, Dressman JB. 2004. Predicting the precipitation of poorly soluble weak bases upon entry in the small intestine. J Pharm Pharmacol 56(1):43-51.

11. Carino SR, Sperry DC, Hawley M. 2006. Relative bioavailability estimation of carbamazepine crystal forms using an artificial stomachduodenum model. J Pharm Sci 95(1):116-125.

12. Carino SR, Sperry DC, Hawley M. 2010. Relative bioavailability of three different solid forms of PNU-141659 as determined with the artificial stomach-duodenum model. J Pharm Sci 99(9):3923-3930.

13. Mudie DM, Amidon GL, Amidon GE. 2010. Physiological parameters for oral delivery and in vitro testing. Mol Pharm 7(5):13881405.

14. Pressman JH, Hofmann AF, Witztum KF, Gertler SL, Steinbach JH, Stokes K, Kelts DG, Stone DM, Jones BR, Dharmsathaphorn K. 1987. Limitations of indirect methods of estimating small bowel transit in man. Dig Dis Sci 32(7):689-699.

15. Hsieh YL, Ilevbare GA, Van Eerdenbrugh B, Box KJ, Sanchez-Felix MV, Taylor LS. 2012. pH-Induced precipitation behavior of weakly ba- sic compounds: Determination of extent and duration of supersaturation using potentiometric titration and correlation to solid state properties. Pharm Res 29(10):2738-2753.

16. Psachoulias D, Vertzoni M, Goumas K, Kalioras V, Beato S, Butler J, Reppas C. 2011. Precipitation in and supersaturation of contents of the upper small intestine after administration of two weak bases to fasted adults. Pharm Res 28(12):3145-3158.

17. Brouwers J, Brewster ME, Augustijns P. 2009. Supersaturating drug delivery systems: The answer to solubility-limited oral bioavailability? J Pharm Sci 98(8):2549-2572.

18. Kim MS, Kim JS, Cho W, Cha KH, Park HJ, Park J, Hwang SJ. 2013. Supersaturatable formulations for the enhanced oral absorption of sirolimus. Int J Pharm 445(1-2):108-116.

19. Kim JS, Mitchell S, Kijek P, Tsume Y, Hilfinger J, Amidon GL. 2006. The suitability of an in situ perfusion model for permeability determinations: Utility for BCS class I biowaiver requests. Mol Pharm 3(6):686-694.

20. Kasim NA, Whitehouse M, Ramachandran C, Bermejo M, Lennernas H, Hussain AS, Junginger HE, Stavchansky SA, Midha KK, Shah VP, Amidon GL. 2004. Molecular properties of WHO essential drugs and provisional biopharmaceutical classification. Mol Pharm 1(1):8596.

21. McFarland JW, Avdeef A, Berger CM, Raevsky OA. 2001. Estimating the water solubilities of crystalline compounds from their chemical structures alone. J Chem Inf Comput Sci. 41 (5):1355-9.

22. Takagi T, Ramachandran C, Bermejo M, Yamashita S, Yu LX, Amidon GL. 2006. A provisional biopharmaceutical classification of the top 200 oral drug products in the United States, Great Britain, Spain, and Japan. Mol Pharm 3(6):631-643.

23. Pade V, Stavchansky S. 1997. Estimation of the relative contribution of the transcellular and paracellular pathway to the transport of passively absorbed drugs in the Caco-2 cell culture model. Pharm Res 14(9):1210-1215.

24. Routledge PA, Shand DG. 1979. Clinical pharmacokinetics of propranolol. Clin Pharmacokinet 4(2):73-90.

25. Taguchi M, Nozawa T, Mizumaki K, Inoue H, Tahara K, Takesono C, Hashimoto Y. 2004. Nonlinear mixed effects model analysis of the pharmacokinetics of metoprolol in routinely treated Japanese patients. Biol Pharm Bull 27(10):1642-1648.

26. Shand DG, Rangno RE. 1972. The disposition of propranolol. I. Elimination during oral absorption in man. Pharmacology 7(3):159 168.

27. Eddington ND, Ashraf M, Augsburger LL, Leslie JL, Fossler MJ, Lesko LJ, Shah VP, Rekhi GS. 1998. Identification of formulation and manufacturing variables that influence in vitro dissolution and in vivo bioavailability of propranolol hydrochloride tablets. Pharm Dev Technol 3(4):535-547.

28. Rekhi GS, Eddington ND, Fossler MJ, Schwartz P, Lesko LJ, Augsburger LL. 1997. Evaluation of in vitro release rate and in vivo absorption characteristics of four metoprolol tartrate immediate-release tablet formulations. Pharm Dev Technol 2(1):11-24.

29. Yamashita S, Kataoka M, Higashino H, Sakuma S, Sakamoto T, Uchimaru H, Tsukikawa H, Shiramoto M, Uchiyama H, Tachiki H, Irie S. 2013. Measurement of drug concentration in the stomach after intragastric administration of drug solution to healthy volunteers: Analysis of intragastric fluid dynamics and drug absorption. Pharm Res 30(4):951-958.

30. Chaudhuri TK, Greenwald AJ, Heading RC. 1975. Measurement of gastric emptying time-A comparative study between nonisotopic aspiration method and new radioisotopic technique. Am J Dig Dis 20(11):1063-1066

31. Steingoetter A, Fox M, Treier R, Weishaupt D, Marincek B, Boesiger P, Fried M, Schwizer W. 2006. Effects of posture on the physiology of gastric emptying: A magnetic resonance imaging study. Scand $\mathrm{J}$ Gastroenterol 41(10):1155-1164. 